\title{
Sevoflurane Inhibits Proliferation and Invasion of Human Ovarian Cancer Cells by Regulating JNK and p38 MAPK Signaling Pathway
}

This article was published in the following Dove Press journal:

Drug Design, Development and Therapy

\section{Kai Kang \\ Yue Wang}

Department of Anesthesiology, Beijing Obstetrics and Gynecology Hospital,

Capital Medical University, Beijing 100026, People's Republic of China
Correspondence: Yue Wang

Department of Anesthesiology, Beijing

Obstetrics and Gynecology Hospital,

Capital Medical University, No. 25I

Yaojiayuan Road, Beijing 100026, People's

Republic of China

Tel +86-1367I077406

Email dryuewang_bj@163.com

\begin{abstract}
Aim: Sevoflurane is a halogen inhaled anesthetic, and we aimed to probe the effect of sevoflurane on proliferation and invasion of human ovarian cancer (OC) and its mechanism. Methods: OC cell lines were divided into 4 groups including control, sevoflurane low concentration (1.7\%), medium concentration (3.4\%) and high concentration $(5.1 \%)$ groups. Flow cytometry and MTT assay were, respectively, employed to detect the cell apoptosis and proliferation. Transwell assay was applied to measure the cell migration and invasion viability. The gene and protein expressions were assessed using qRT-PCR and Western blot. The expressions of MAPK signaling pathway-related proteins were evaluated by Western blot. The p38 and JNK inhibitors were, respectively, added into the high concentration group to analyze the relationship between sevoflurane and modulatingmitogen-activated protein kinase (MAPK) pathway in OC. Nude mice models were constructed to explore the effect of sevoflurane on OC tumor growth in vivo.

Results: Sevoflurane inhibited OC proliferation in vitro and in vivo. It could also promote OC cell apoptosis in a dose-dependent manner. Sevoflurane suppressed the OC cell migration and invasion, and these effects were positively correlated with the dose of sevoflurane. Moreover, sevoflurane treatment inhibited the expressions of PCNA, Twist, cleavedcaspase-3/caspase-3, MMP-2 and MMP-9. In addition, sevoflurane repressed the phosphorylation of JNK and p38 MAPK. When the MAPK pathway was interdicted, the cell proliferation, apoptosis, migration and invasion activity were recovered after sevoflurane treatment.
\end{abstract}

Conclusion: Sevoflurane affected cell biological activities in OC by regulating JNK and p38 MAPK signaling pathway.

Keywords: ovarian cancer, sevoflurane, proliferation, migration and invasion, MAPK signaling pathway

\section{Introduction}

Ovarian cancer (OC), which has an obscure onset and lacks early clinical symptoms, is common in perimenopausal women. ${ }^{1}$ The incidence of OC is the third highest among all gynecological tumors, after cervical cancer and endometrial cancer, but its mortality rate is the first. ${ }^{2}$ Worldwide, there were 300,000 new OC cases in 2018 and 200,000 deaths from OC. ${ }^{3}$ In China, 52,100 new cases of OC and 22,500 deaths were reported in $2016 .{ }^{4}$ Unlike other tumors, OC tumors tend to invade and metastasize within the peritoneum, rarely through blood vessels or lymph nodes. ${ }^{2}$ Currently, the clinical treatment strategies for OC are very limited, 
mainly including surgery, chemotherapy and chemotherapeutic drugs. ${ }^{5}$ Many OC cases were found at the advanced stage of extensive metastasis, more than $75 \%$ of the women at the time of diagnosis is to late stage (III or IV). Half of the OC patients relapsed within 16 months, and the 5-year overall survival rate was less than $50 \%$, while the 5 -year survival rate for early-stage OC was also very low. ${ }^{6}$ The main reasons for the poor diagnosis and prognosis of OC are that OC usually presents asymptomatic characteristics in the early stage and easy to develop resistance to traditional platinum chemotherapy drugs. ${ }^{7}$

For the past three decades, the standard treatment for OC has been tumor cell ablation, supplemented by taxoid-based and platinum-based chemotherapy. ${ }^{8}$ Even after standard treatment, most patients showed resistance to chemotherapy and relapsed. Chemotherapy-resistant patients were often treated with second-line or higher chemotherapy agents such as topotecan, gemcitabine, liposomal doxorubicin, and etoposide. Unfortunately, the progression-free survival of these patients was only 2-4 months, and the overall survival was $10-14$ months. ${ }^{9}$ Therefore, finding more effective treatment or drugs to inhibit OC was helpful to reduce the mortality of OC and improved the survival status of patients. Sevoflurane is a commonly used inhalation anesthetic drug. Previous studies have shown that sevoflurane could play a role in a variety of tumors, such as colon cancer, ${ }^{10}$ breast cancer, ${ }^{11}$ lung cancer ${ }^{12}$ and cervical cancer. ${ }^{13}$ These reports suggested that sevoflurane had potential clinical application value in malignant tumor, but its effect on malignant behavior of OC cells and mechanism had not been completely clarified.

In the current study, we explored the effect of sevoflurane treatment on OC cells and its functional mechanism. We investigated that sevoflurane inhibited the OC cell lines (SKOV3 and OVCAR3) proliferation in vitro and in vivo. And the OC cell apoptosis was enhanced after sevoflurane treatment. Sevoflurane could also decrease the OC cells migration and invasion viability. All of these effects of sevoflurane on $\mathrm{OC}$ cell lines were positively correlated with the concentration of sevoflurane. In addition, we demonstrated that sevoflurane treatment effected on OC cells via modulatingmitogen-activated protein kinase (MAPK) signaling pathway. These findings suggested that sevoflurane might be a potential and novel agent in OC therapy.

\section{Materials and Methods Cell Culture}

Human OC cell lines OVCAR3 and SKOV3, which were provided by Shanghai Cell Bank, Chinese Academy of Sciences (Shanghai, China), were used in this study. OC cell lines SKOV3 and OVCAR3 were cultured into DMEM medium with $10 \%$ fetal bovine serum (FBS, Invitrogen Carlsbad, CA, USA), and the culture environment was $37^{\circ} \mathrm{C}$ and $5 \% \mathrm{CO}_{2}$.

\section{Protocol of OC Cells Exposure to Sevoflurane}

Sevoflurane treatment of cells referred to previous studies. $^{14,15}$ In brief, cell culture plates were placed in a sterile and closed container with inlet and outlet connectors. An anesthesia machine (GE Healthcare Life Sciences, Chalfont, UK) was connected to the inlet port, and attached to a sevoflurane vaporizer (Sevorane; Abbott, Abbot Park, IL), which was used to supply sevoflurane gas (mixed with $95 \% \mathrm{O}_{2} / 5 \% \mathrm{CO}_{2}$ ) into the container. The concentrations of sevoflurane in the container were monitored by a gas monitor (PM8060, Drager, Lübeck, Germany), which was connected to the outlet port of container. The cell lines were exposed to different concentrations $(1.7 \% \mathrm{v} / \mathrm{v}, 3.4 \% \mathrm{v} / \mathrm{v}$ or $5.1 \% \mathrm{v} / \mathrm{v})$ of sevoflurane for $6 \mathrm{~h}$, and, respectively, divided into 4 groups: Control group, high sevoflurane concentration group (5.1\% group), medium sevoflurane concentration group (3.4\% group) and low sevoflurane concentration group (1.7\% group). The cells of the control group only treated by a mixture of $95 \% \mathrm{O}_{2}+5 \% \mathrm{CO}_{2}$. Then, the cells were cultured in normal conditions for $24 \mathrm{~h}$ before further analyses.

\section{MAPK Signaling Pathway Inhibitor Treatment}

To block MAPK signaling pathway, the SKOV3 cell lines were incubated with p38 inhibitor SB203580 (20 $\mu \mathrm{M}$, ab120162, Abcam, Cambridge, MA, USA) or JNK inhibitor SP600125 (10 $\mu \mathrm{M}$, ab120065, Abcam) according to the manufacturer's instructions. Briefly, the confluent SKOV3 cell lines were pre-incubated for $8 \mathrm{~h}$ with SB203580 or SP600125 prior to exposure to $5.1 \%$ sevoflurane for $4 \mathrm{~h}$. Then, the cells were divided into 4 groups: control group, $5.1 \%$ group, $5.1 \%+\mathrm{p} 38$ inhibitor group $(5.1 \%+\mathrm{SB} 203580$ group $)$ and $5.1 \%+\mathrm{JNK}$ inhibitor group (5.1\% + SP600125 group). The cell proliferation, apoptosis, migrations and invasion were detected at 
$24 \mathrm{~h}$ post-sevoflurane treatment. Each treatment was performed in triplicate.

\section{3-(4,5-Dimethyl-2-Thiazolyl)- 2,5-Diphenyl-2-H-Tetrazolium Bromide (MTT) Assay}

The cell proliferation activity was measured using MTT assay. After sevoflurane treatment for $24 \mathrm{~h}$, cells were seeded into 96-well plates with a dose of 5000 cells for one well. Briefly, $10 \mu \mathrm{L}$ of MTT solution $(5 \mathrm{mg} / \mathrm{mL}$, Thermo Fisher, Wilmington, DE, USA) was used to incubate cells for $4 \mathrm{~h}$, and then $200 \mu \mathrm{L}$ of dimethylsulfoxide (DMSO, Thermo Fisher) was added into each well for $10 \mathrm{~min}$. Absorbance was evaluated at $490 \mathrm{~nm}$ using a microporous plate spectrophotometer (Thermo Labsystems, Vantaa, Finland). MTT assay was repeated 3 times.

\section{Cell Apoptosis}

The cell apoptosis was detected using flow cytometry. After sevoflurane treatment for $24 \mathrm{~h}$, cell lines were digested and collected by trypsinase (Invitrogen). The cells were washed with precooled PBS (Invitrogen) for 2 times, and $1 \times 10^{6}$ cells $/ \mathrm{mL}$ cell suspension was prepared. Cell suspensions (100 $\mu \mathrm{L} /$ tube) were stained by propidium iodide (Nanjing KeyGen Biotech, Nanjing, China) and fluorescein isothiocyanate-conjugated Annexin V (Nanjing KeyGen Biotech) according to the instructions. The cells were gently mixed and placed in dark for 15 mins. The results were determined by flow cytometry (BD Biosciences, NJ, USA) within 1 h. Flow cytometry assay was repeated 3 times.

\section{Transwell Assay}

The cell migration and invasion viability were both measured by transwell assay. For migration viability, FBS-free DMEM medium was used to resuspend cells. The concentration of cells was adjusted to $1 \times 10^{6}$ cells $/ \mathrm{mL}$. $200 \mu \mathrm{L}$ cell suspension was added to each chamber. A total of $600 \mu \mathrm{L}$ of DMEM medium (10\% FBS) was added to each pore in the lower chamber. After $24 \mathrm{~h}$ of incubation, the cells were removed from the chamber and wiped off with cotton swabs. The cells were stained with $0.1 \%$ crystal violet for 20 mins. $200 \times$ field microscope images were taken (5 fields/group). Migration inhibition rate $=(1-$ number of migrating cells in experimental group/number of migrating cells in control group) $\times 100 \%$. For invasion viability, each transwell cell was coated with $50 \mu \mathrm{L}$ Matrigel matrix gel (BD Biosciences, USA) for $24 \mathrm{~h}$. The other operations were the same as the migration experiment. Invasion inhibition rate $=(1-$ number of migrating cells in experimental group/number of migrating cells in control group) $\times 100 \%$. All experiments were repeated 3 times.

\section{Western Blot}

Radio Immunoprecipitation Assay (RIPA) lysis buffer, provided by Sigma (St. Louis, MO, USA), was used to collect protein lysates. BCA method was applied to quantify the total protein concentration. Proteins were added to SDS polyacrylamide gel electroelectrometry (PAGE), and electrotransferred to polyvinylidene fluoride (PVDF) membrane after electrophoresis separation. Blocking buffer ( $5 \%$ bovine serum albumin, BSA) was used to block the membranes at room temperature. After blocking, primary antibodies were used to incubate the membranes overnight at $4^{\circ} \mathrm{C}$. Primary antibodies (Abcam, Cambridge, MA, USA) were used at the following dilutions: anti-PCNA (1:1000, ab18197), anti-cleaved-caspase -3 (1:1000, ab2302), anti-caspase-3 (1:500, ab13847), antiTwist (1:2000, ab175430), anti-MMP-2 (1:1000, ab37150), anti-MMP-9 (1:1000, ab73734), antiphosphorylation-p38 (p-p38) (1:1000, ab4822), anti-p38 (1:1000, ab31828), anti-p-JNK (1:1000, ab124956), antiJNK (1:1000, ab179461), anti-p-ERK (1:1000, ab65142), anti-ERK (1:10,000, ab32537), anti-GAPDH (1:500, ab8245). After washing the membranes Tris-buffered saline containing $0.1 \%$ Tween-20 (TBS-T) for 4 times, the secondary antibody was added and incubated at $37^{\circ} \mathrm{C}$ for $30 \mathrm{~min}$. GAPDH served as the loading control. All experiments were repeated 3 times.

\section{RNA Extraction and Real-Time Quantitative Polymerase Chain Reaction (qRT-PCR)}

Trizol kits (Invitrogen) were used to extract the total RNA from human OC cell lines, the RNA reverse transcription with RT MasterMix PrimeScript ${ }^{\mathrm{TM}}$ kit for cDNA (TaKaRa, Dalian, China). qRT-PCR was proceeded with SYBR Premix Ex Taq $^{\mathrm{TM}}$ II kit (TaKaRa) using cDNA as a template according to the manufacturer's instruction, GAPDH was used as the internal control. Relative quantitative method was used to determine the results and $2^{-\triangle \triangle \mathrm{Ct}}$ was calculated. The experiment was repeated for three times. The primer sequences used in this study were as follows: PCNA forward primers 5'-GCCTGAC AAATGCTTGCT-3', reverse primers 5'-GCGGGAAGGA 
GGAAAG-3'; caspase-3 forward primers 5'-CAGTGATG CTGTGCTATGAAT-3', reverse primers 5'-CAGATGCCT AAGTTCTTCCAC-3'; Twist forward primers 5'-AGCC TGAGCAACAGCGA-3', reverse primers 5'-ACAGCCC GCAGACTTCTT-3'; MMP-2 forward primers 5'-CGCC TTTAACTGGAGCAAA-3', reverse primers 5'-AGGTTA TCGGGGATGGC-3'; MMP-9 forward primers 5'-ACG CAGACATCGTCATCC-3', reverse primers 5'-CCAGG GACCACAACTCG-3'; GAPDH forward primers 5'-CCT TCCGTGTCCCCACT-3', forward primers 5'-GCCTGCT TCACCACCTTC-3'. GAPDH served as the internal control. All experiments were repeated 3 times.

\section{Xenograft Tumor Growth Assay}

Female BALB/c nude mice (4-6 weeks; $n=6$ /group) were purchased from Shanghai Laboratory Animal Center (Shanghai, China) and raised in specific pathogen-free conditions. SKOV3 cell lines were incubated for $24 \mathrm{~h}$ after gas treatment with or without $5.1 \%$ sevoflurane stimulation. Then, the SKOV3 cell lines were prepared as a $2 \times 10^{6}$ cells $/ \mathrm{mL}$ single-cell suspension. The female BALB/c nude mice were hypodermic injected with the cell suspension $(0.2 \mathrm{~mL} / \mathrm{mouse})$ and divided into 2 groups: Control group and 5.1\% (sevoflurane) group. The tumor volume was measured every 3 days, and the tumor volume (V) was calculated according to the formula: $\mathrm{V}=1 / 2 \mathrm{ab}^{2}\left(\mathrm{~mm}^{3}\right)$. After 3 weeks, the mice were killed by air embolization and the tumor weight was calculated. The use and care of animals was carried out under the guidelines approved by the Institutional Animal Care and Use Committee of Beijing Obstetrics and Gynecology Hospital, Capital Medical University.

\section{Statistical Analysis}

SPSS 19.0 was processed for statistical analysis. The measurement data were expressed as mean \pm standard deviation (SD). Student's $t$-test and one-way ANOVA were, respectively, used to compare the differences of two independent samples and multiple groups. $P<0.05$ was considered statistically significant.

\section{Result}

\section{Sevoflurane Inhibited Proliferation and} Promoted Apoptosis in OC Cell Lines

At the beginning of this study, the OC cell lines SKOV3 and OVCAR3 were both treated without or with different concentrations of sevoflurane $(1.7 \%, 3.4 \%$ or $5.1 \%)$. Then, the cell proliferation activity of SKOV3 and OVCAR3 was determined using MTT assay. The data from MTT assay exhibited that sevoflurane treatment significantly inhibited the cell proliferation in SKOV3 and OVCAR3 cell lines compared to the control group $(P<0.01$, Figure 1A). And the inhibitory effect of sevoflurane on OC cells proliferation was dose-dependent (Figure 1A). We also checked the effect of sevoflurane treatment on OC cell apoptosis and found that sevoflurane notably enhanced the SKOV3 and OVCAR3 cells apoptosis compared with the control group on a dose-dependent $(P<0.01$, Figure 1B). Above data investigated that sevoflurane treatment could inhibit proliferation and promote apoptosis in OC cells.

\section{Sevoflurane Inhibited Migration and Invasion in OC Cell Lines}

To further explore the influence of sevoflurane on OC cell lines activities, transwell assay was employed. In SKOV3 cell lines, the results from transwell assay showed that cell migration and invasion activities were both dramatically decreased in sevoflurane groups compared to that in the control group ( $P<0.01$, Figure 2$)$. Likely, sevoflurane also obviously reduced OVCAR3 cells migration and invasion compared with the control group ( $P<0.01$, Figure 2$)$. All of these effects of sevoflurane on SKOV3 and OVCAR3 cells were in positive correlation with sevoflurane concentration. These findings confirmed that sevoflurane suppressed OC cell migration and invasion viability.

\section{Effect of Sevoflurane on Proteins Expressions of Human OC Cell Proliferation and Invasion}

To define the effects of sevoflurane on OC cells proliferation, apoptosis and invasion, the mRNA expression levels of proliferation-related factor (PCNA), apoptosis-related factor (caspase-3) and invasion-related factors (Twist, MMP-2 and MMP-9) were determined using qRT-PCR. As shown as Figure 3A, PCNA, Twist, MMP-2 and MMP9 mRNA expressions were significantly decreased while caspase-3 expression was markedly increased in sevoflurane groups compared to that in the control group $(P<0.01)$. Moreover, the protein expressions of PCNA, Twist, cleaved-caspase-3/caspase-3, MMP-2 and MMP-9 were assessed by Western blot. Similar to the results of qRT-PCR, PCNA, Twist, MMP-2 and MMP-9 expressions were inhibited as well as cleaved-caspase-3/caspase-3 expression was accelerated after sevoflurane treatment (Figure 3B). And the effects of sevoflurane on these 

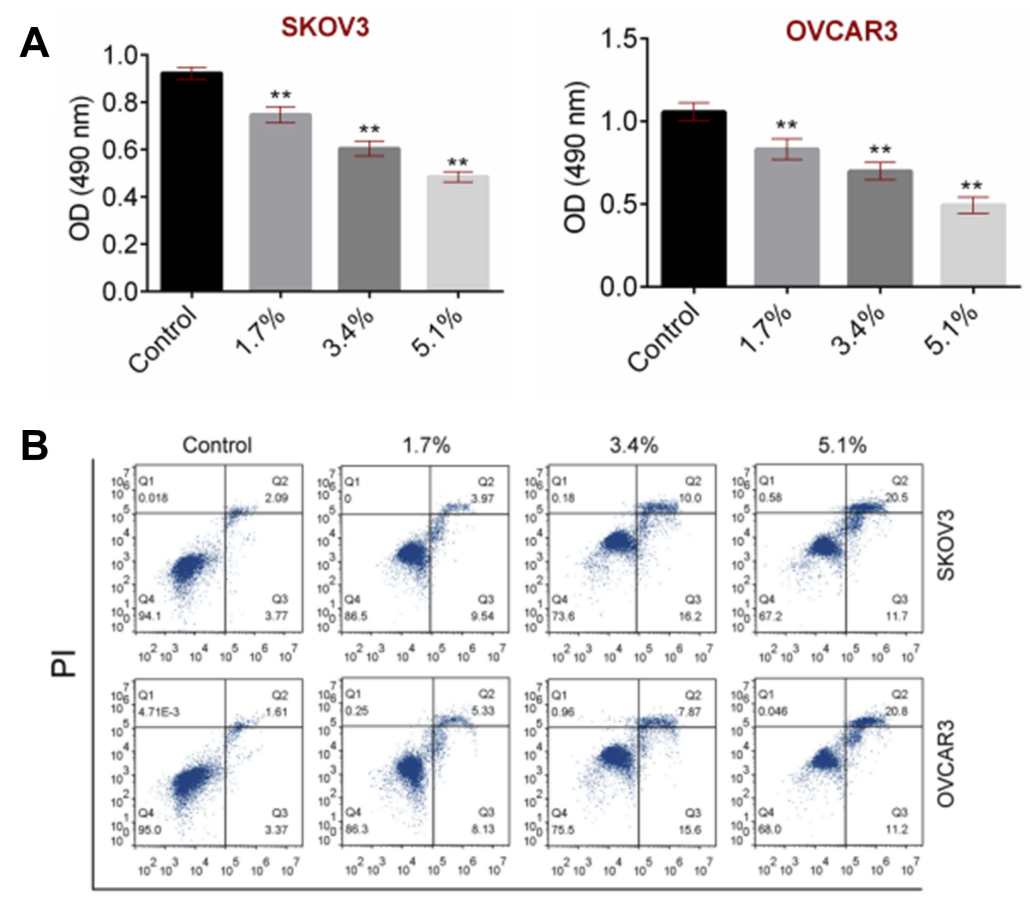

FITC

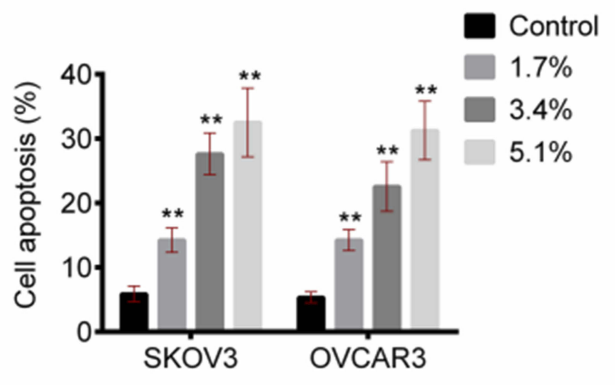

Figure I Sevoflurane inhibited proliferation and promoted apoptosis in OC cell lines SKOV3 and OVCAR3. SKOV3 and OVCAR3 were both treated with various concentrations of sevoflurane and divided into 4 groups contained Control group, 1.7\% (sevoflurane) group, 3.4\% (sevoflurane) group and 5. 1\% (sevoflurane) group. (A) The cell proliferation activity of SKOV3 and OVCAR3 cell lines was detected using MTT assay. (B) The cell apoptosis of SKOV3 and OVCAR3 cell lines was determined using flow cytometry. Data were shown as mean $\pm \mathrm{SD}$. $* * P<0.01$ vs Control group.

factors' expressions were positively related to the dose of sevoflurane. These results suggested that sevofluranerepressed cell proliferation, migration and invasion and promoted apoptosis in OC.

\section{Sevoflurane Inhibited Mitogen-Activated Protein Kinase (MAPK) Signaling Pathway}

To analyze the potential mechanism of sevoflurane effecting on OC cells, the expression levels of MAPK signaling pathway-related factors, including p-p38, p38, p-JNK, JNK, p-ERK and ERK, were detected via Western blot. Figure 4 displays that the expression of p-p38/p38 and $\mathrm{p}-\mathrm{JNK} / \mathrm{JNK}$ was both remarkably suppressed on a dosedependent manner in SKOV3 and OVCAR3 cell lines of sevoflurane groups compared to that of the control group $(P<0.01)$. Although sevoflurane significantly inhibited the expression of ERK in the $5.1 \%$ group, the effect of sevoflurane on the ERK expression was obviously lower than that on the p38 or JNK expressions in the $1.7 \%$ and $3.4 \%$ groups. These data elucidated that sevoflurane interdicted the phosphorylation of p38 and JNK of MAPK signaling pathway.

\section{Sevoflurane Regulated the Development of OC by Targeting MAPK Signaling}

\section{Pathway}

To identify the functional mechanism of sevoflurane in OC, MAPK signaling pathway was inhibited by p38 inhibitor (SB203580) or JNK inhibitor (SP600125). SKOV3 cell lines (5.1\% group) were used to proceed with this experiment and the cell activities were examined. The data from MTT assay showed that the proliferation activities in $5.1 \%+\mathrm{SB} 203580$ and $5.1 \%+\mathrm{SP} 600125$ groups of OC cells were significantly higher than that in $5.1 \%$ group $(P<0.01$, Figure 5A). After sevoflurane treating, the cell apoptosis of the 4 groups was detected using flow cytometry and we investigated that SB203580 or SP600125 treatments obviously recovered the cell apoptosis induced by sevoflurane in OC cell lines SKOV3 $(P<0.01$, Figure 5B). Furthermore, the migration and invasion of SKOV3 cells were evaluated through transwell assay. Figure 5C exhibits that both SB203580 and SP600125 treatments could partly abolish the decrease of migration and invasion which are induced by sevoflurane in OC cell lines. These results illustrated that sevoflurane effected the 

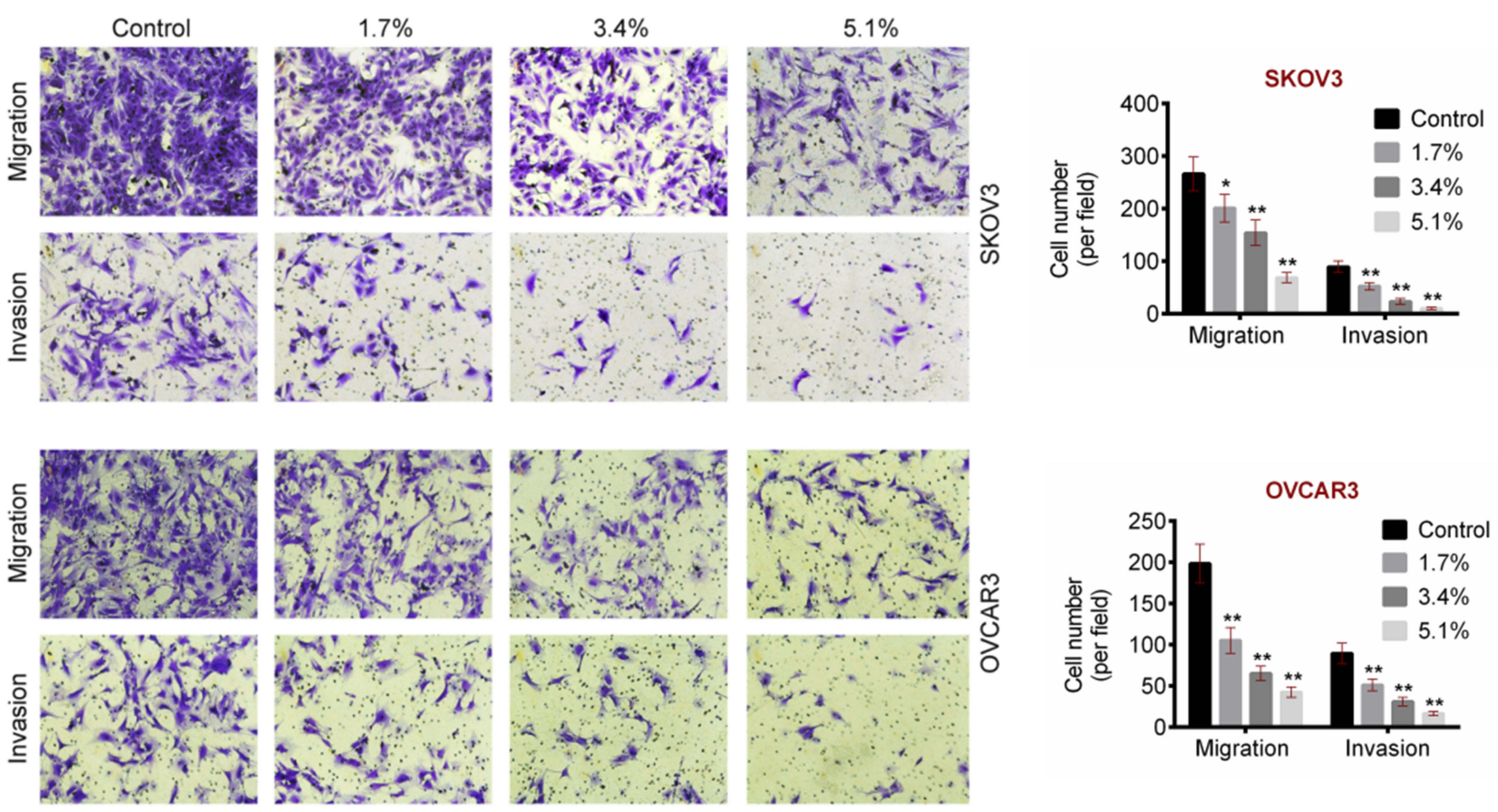

Figure 2 Sevoflurane inhibited migration and invasion in OC cell lines SKOV3 and OVCAR3. The cell migration and invasion activity were detected using transwell assay. Data were shown as mean \pm SD. $* P<0.05$, $* * P<0.01$ vs Control group.

proliferation, apoptosis, migration and invasion of OC cell lines via regulating the MAPK signaling pathway.

\section{Sevoflurane Inhibited OC Tumor Growth in vivo}

To explore the effect of sevoflurane on OC in vivo, xenograft tumor growth assay was performed. OC cell lines SKOV3 with or without $5.1 \%$ sevoflurane treatment were injected into nude mice. As shown in Figure 6A, the tumor volume of $5.1 \%$ group was significantly smaller than that of the control group $(P<0.01)$. Furthermore, the tumor size and weight of mice in $5.1 \%$ group were both less than that in control group $(P<0.01$, Figure $6 \mathrm{~B}$ and $\mathrm{C})$. These data suggested that sevoflurane treatment could inhibit $\mathrm{OC}$ tumor growth in vivo.

\section{Discussion}

$\mathrm{OC}$ is one of the most common malignant tumors in female reproductive system. ${ }^{16}$ In recent years, the incidence of $\mathrm{OC}$ has been increasing year by year. The location of $\mathrm{OC}$ is hidden, which is the main cause of the death of gynecological malignant tumors. At present, the early diagnosis of $\mathrm{OC}$ is still a worldwide problem. Most patients with $\mathrm{OC}$ cannot be diagnosed until the advanced stage of the disease. ${ }^{17}$ Mortality and recurrence rate of OC patients are high. Platinum chemotherapeutic drugs are the first choice for OC treatment, it binds with DNA to form intra-and/or inter-chain adducts, which prevents the normal synthesis and replication of DNA, cause damage to genetic materials, induce cancer cell death and play an anti-tumor role. ${ }^{18}$ At present, the basic treatment principle of OC is extensive cytoreductive surgery combined with cisplatin and paclitaxel chemotherapy. ${ }^{19}$ However, OC is prone to chemotherapy resistance and recurrence of cancer focus. The recurrence rate of this scheme is as high as $70 \%$ in 2 years after the operation. ${ }^{20}$ In recent years, the exploration of effective new drugs for the treatment of OC has become the focus of OC research. Fu et $\mathrm{al}^{21}$ reported that metformin, an antidiabetic drug, could alleviate OC cell proliferation via suppressing $\mathrm{m}$-TOR/PI3K/ Akt pathway and inducing G2/M cell cycle interdict. Xu et $\mathrm{al}^{22}$ confirmed that Dihydromyricetin, a natural product which was isolated from Ampelopsis grossedentata, could inverse multi-drug resistance against $\mathrm{OC}$ cells and promote cell apoptosis by inhibiting survivin expression. However, the clinical diagnosis and treatment of $\mathrm{OC}$ are still difficult. Therefore, there is an urgent need to find effective new drugs for the treatment of $\mathrm{OC}$, which is of great significance to improve the quality of life and survival rate of patients with OC.

Sevoflurane was usually well known as a common anesthetic in clinical practice. ${ }^{23}$ In recent years, scholars 
A
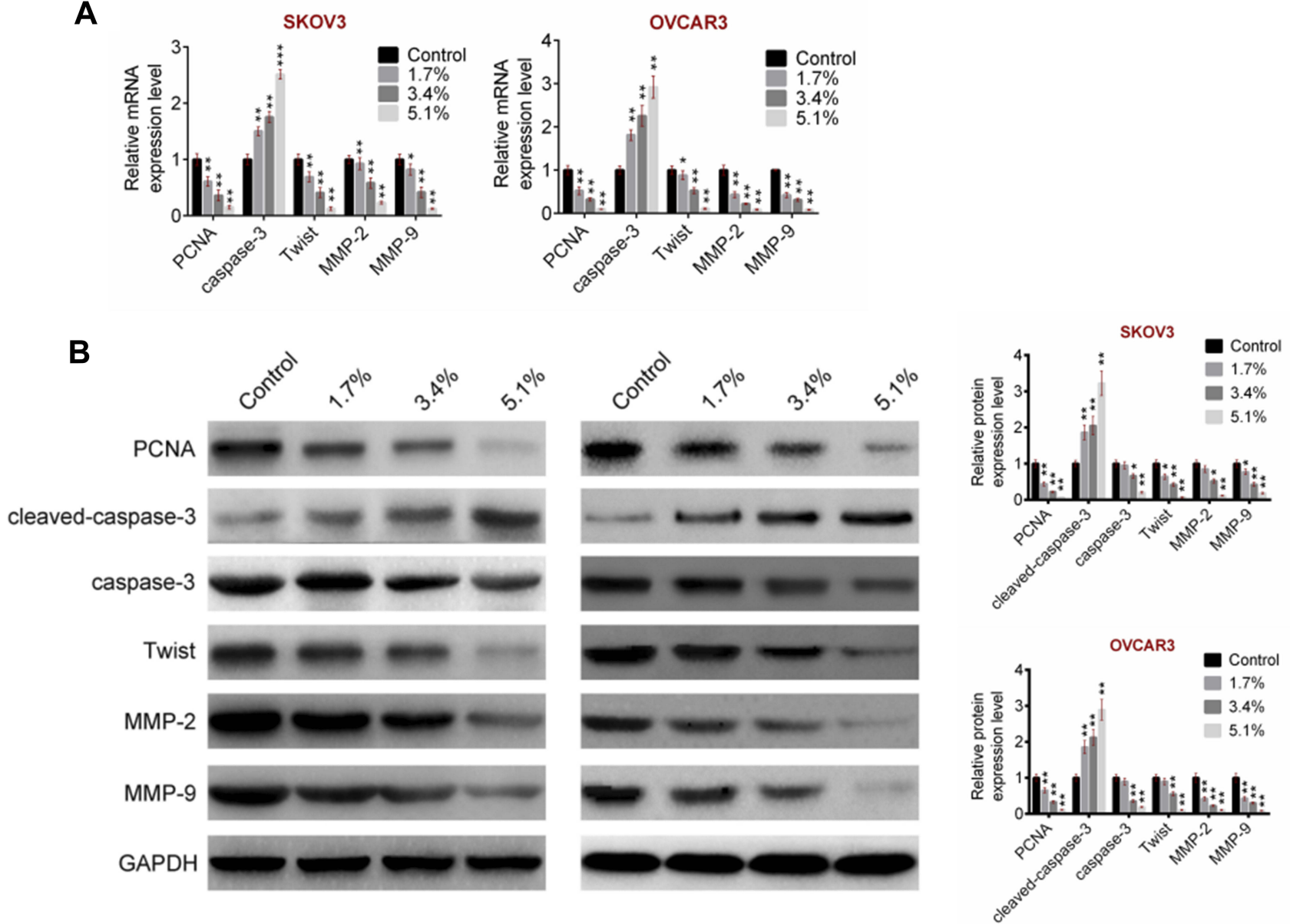

Figure 3 Sevoflurane inhibited proliferation-related and invasion-related genes expressions and promoted apoptosis-related genes expressions in SKOV3 and OVCAR3 cell lines. (A) The mRNA of proliferation-related gene (PCNA), invasion-related genes (Twist, MMP-2 and MMP-9) and apoptosis-related gene (caspase-3) expressions were detected using qRT-PCR. (B) The protein expressions of PCNA, cleaved-caspase-3/caspase-3, Twist, MMP-2 and MMP-9 were determined using Western blot. Data were shown as mean $\pm S D$. $* P<0.05, * * P<0.01, * * * P<0.001$ vs Control group.

have gradually understood the therapeutic effects of sevoflurane in various tumors. Liu et $\mathrm{al}^{24}$ found that sevoflurane inhibited breast cancer cell proliferation by triggering cell G1 phase arrest and promoting the expression level of miR-203. Yang et $\mathrm{al}^{25}$ treated head and neck squamous cell carcinoma (HNSCC) cell lines FaDu and CAL-27 with sevoflurane and proved that sevoflurane-repressed cell viability by modulating HIF-1 $\alpha$ signaling pathway in HNSCC cells. Liang et $\mathrm{al}^{26}$ reported that an induction of cell apoptosis and a suppression of cell proliferation were observed in lung cancer cells which were treated with sevoflurane, and cell cycle arrest was also occurred. In the previous study, volatile anaesthetics were reported to be related to the expression of metastatic proteins and genes in OC, and inhalational anaesthetics might have a profound and distinct effect on $\mathrm{OC}$ tumor growth. ${ }^{27}$ However, the role and mechanism of sevoflurane in OC are still unclear. In the current study, we treated SKOV3 and OVCAR3 cell lines with different concentrations of sevoflurane, and found that sevoflurane significantly inhibited cell proliferation, migration and invasion and induced apoptosis of the OC cell lines. And, the effects of sevoflurane on OC cells were all positively correlated with the dose of sevoflurane. Moreover, we also proved that sevoflurane treatment suppressed OC tumor growth in vivo.

PCNA and caspase- 3 were, respectively, reported to be related to cell proliferation and apoptosis ${ }^{28,29}$ as well as Twist, ${ }^{30}$ MMP- $2^{31}$ and MMP- $9^{32}$ were identified as cell invasion-related factors. In this study, the expression levels of PCNA, caspase-3, Twist, MMP-2 and MMP-9 were detected by qRT-PCR and Western blot. Data from qRT-PCR and Western blot showed that sevoflurane could suppress the PCNA, Twist, MMP-2 and MMP-9 expression and enhanced the cleaved-caspase-3/caspase-3 


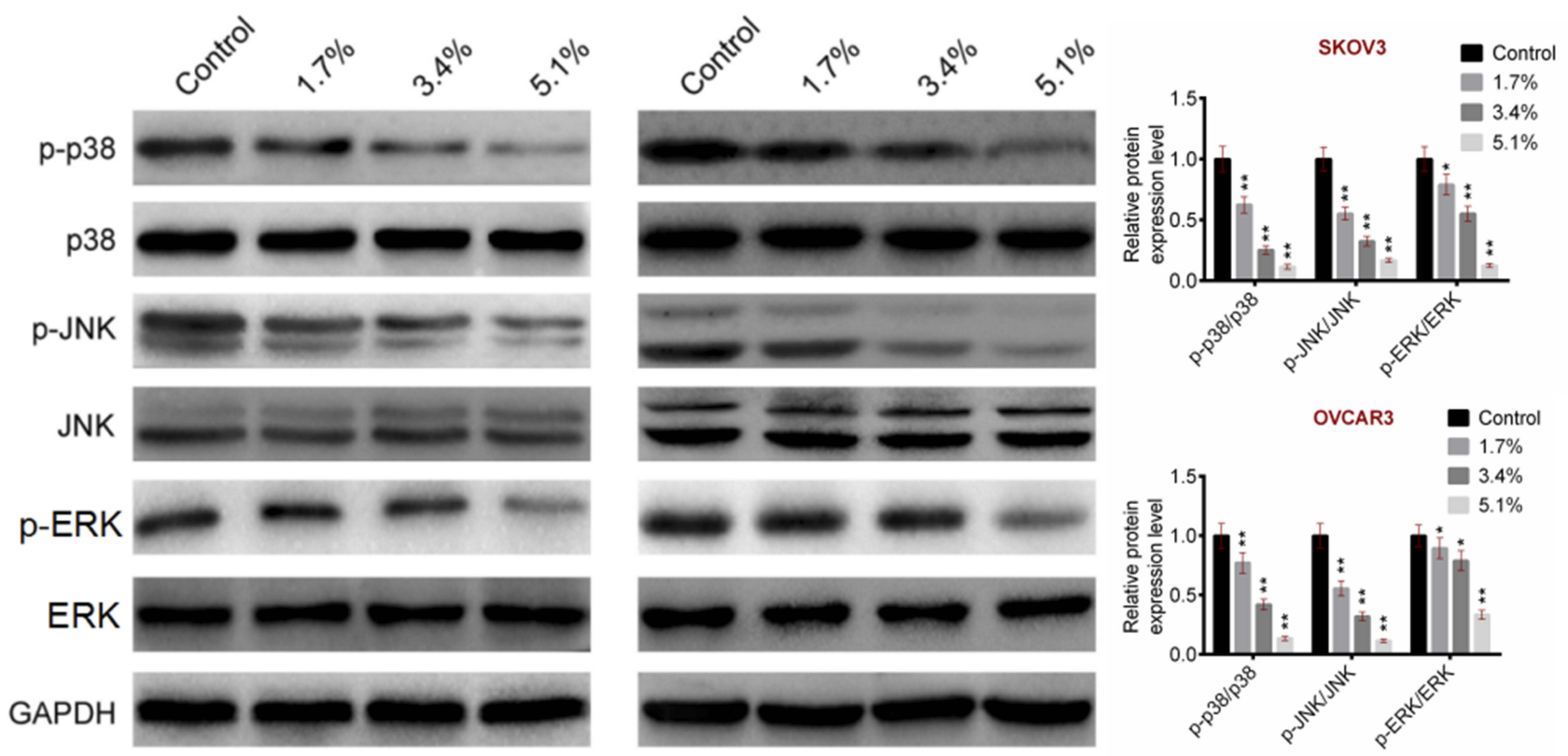

Figure 4 Sevoflurane inhibited MAPK pathway-related proteins expressions. The protein expressions of MAPK pathway-related proteins (p-p38/p38, p-JNK/JNK and p-ERK/ ERK) were detected using Western blot. Data were shown as mean $\pm S D$. $* P<0.05, * * P<0.01$ vs Control group.

A

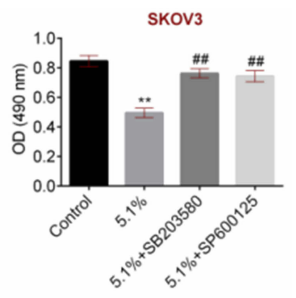

C

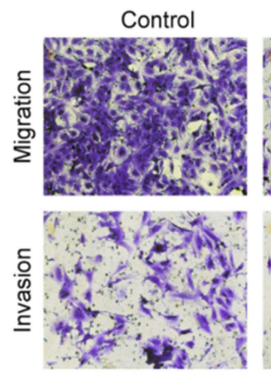

B

$\bar{\alpha}$

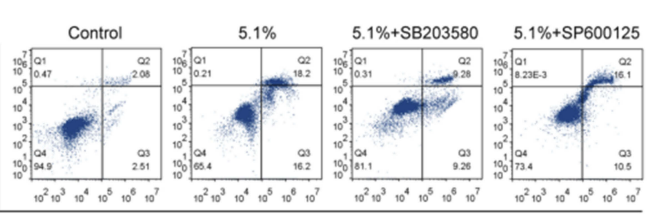

FITC

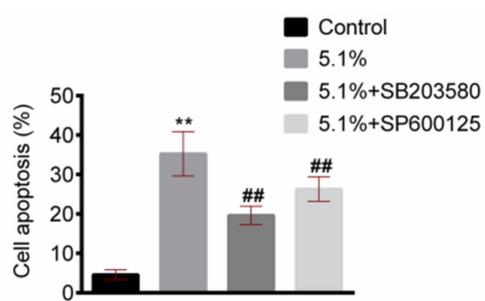

$5.1 \%$
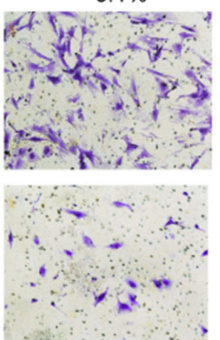

$5.1 \%+$ SB2 203580
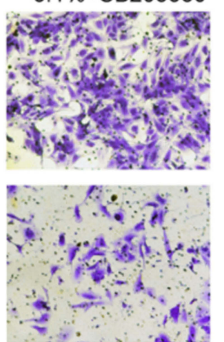

$5.1 \%+$ SP 600125

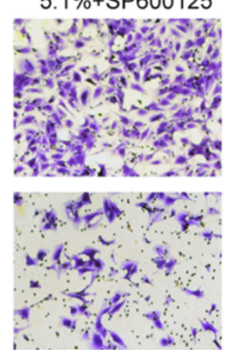

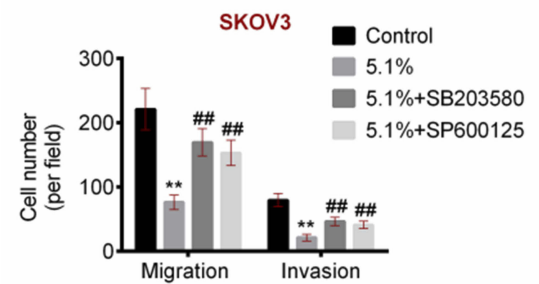

Figure 5 Sevoflurane targeted MAPK signaling pathway to regulate OC cells development. SKOV3 (with $5.1 \%$ sevoflurane) cell lines were treated with p38 inhibitor (SB203580) or JNK inhibitor (SP600 I25) and divided into 4 groups included Control group, 5.1\% (sevoflurane) group, $5.1 \%+$ SB203580 group and 5. 1\% + SP600 I25 group. (A) The cell proliferation activity of SKOV3 cell lines was detected using MTT assay. (B) The cell apoptosis of SKOV3 cell lines was determined using flow cytometry. (C) The cell migration and invasion of SKOV3 cell lines were measured using transwell assay. Data were shown as mean \pm SD. $* * P<0.01$ vs Control group, ${ }^{\#} P<0.01$ vs $5.1 \%$ (sevoflurane) group.

expression. It also indicated that sevoflurane-repressed proliferation and invasion and induced apoptosis in $\mathrm{OC}$ cell lines.

MAPK is an important pathway of intracellular signal transduction, an intersection point and common pathway of multiple signal transduction, which is widely distributed in the cytoplasm and transmits extracellular stimulus signals to the nucleus, and it can regulate of cell proliferation, differentiation and stress. ${ }^{33}$ P38 and JNK were reported to have belonged to principal MAPK pathways, ${ }^{34,35}$ and the expressions of $\mathrm{p} 38$ and JNK were assessed in our study. Dramatically decreases of p-p38/p38 and $\mathrm{p}-\mathrm{JNK} / \mathrm{JNK}$ expressions were observed in OC cells of sevoflurane groups compared to that of the control group. 
A

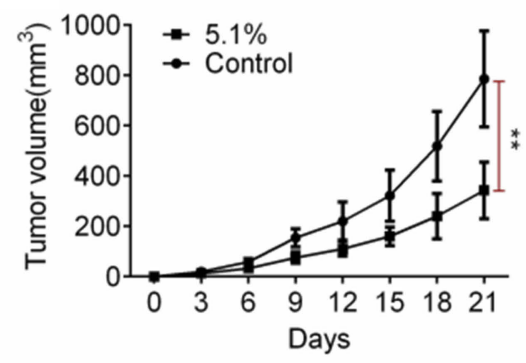

B

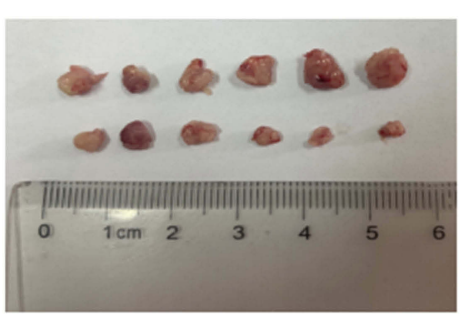

C

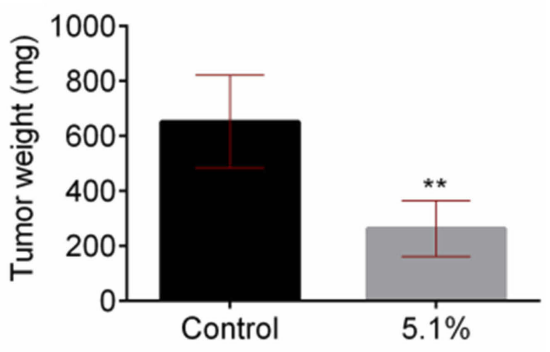

Figure 6 Sevoflurane inhibited OC tumor growth in vivo. (A) The tumor volume was measured after every 3 days. (B) The tumor size of OC mice model was measured after the mice were killed. (C) The tumor weight of $O C$ mice model was measured after the mice were killed. Data were shown as mean \pm SD. $* * P<0.0$ I vs Control group.

To verify whether sevoflurane affected OC cell lines through modulating MAPK pathway, p38 inhibitor SB203580 and JNK inhibitor SP600125 were used to treat SKOV3 cells with $5.1 \%$ sevoflurane. After that, SB203580 and SP600125 treatments both markedly accelerated proliferation, migration and invasion and blocked apoptosis in SKOV3 cells compared to the sevoflurane group. These results suggested that the therapeutic effect of sevoflurane on OC was significantly reduced when the MAPK pathway was blocked. Therefore, sevofluraneregulated cell phenotype of OC cells via p38 and JNK MAPK signaling pathway.

In conclusion, we demonstrated that sevofluraneinhibited proliferation, migration and invasion as well as facilitated apoptosis on a dose-dependent manner in OC cell lines via regulating p38 and JNK MAPK signaling pathway. It provided a novel strategy for the treatment of OC and a theoretical basis for the application of sevoflurane in OC therapy. However, the research of the potential influence of sevoflurane on OC biology is just beginning. Before making any decision to change the current clinical practice, a large number of preclinical and clinical studies are needed.

\section{Availability of data and materials}

All data generated and/or analyzed during this study are included in this published article.

\section{Author Contributions}

Both authors contributed to data analysis, drafting and revising the article, gave final approval of the version to be published, and agree to be accountable for all aspects of the work.

\section{Disclosure}

The authors declare that they have no competing interests.

\section{References}

1. Lengyel E. Ovarian cancer development and metastasis. Am J Pathol. 2010;177(3):1053-1064. doi:10.2353/ajpath.2010.100105

2. Jayson GC, Kohn EC, Kitchener HC, et al. Ovarian cancer. Lancet. 2014;384(9951):1376-1388. doi:10.1016/S0140-6736(13)62146-7

3. Bray F, Ferlay J, Soerjomataram I, et al. Global cancer statistics 2018: GLOBOCAN estimates of incidence and mortality worldwide for 36 cancers in 185 countries. CA Cancer J Clin. 2018;68 (6):394-424. doi:10.3322/caac.21492

4. Siegel RL, Miller KD, Jemal A. Cancer statistics, 2016. CA Cancer J Clin. 2016;66(1):7-30. doi:10.3322/caac.21332

5. Matulonis UA, Sood AK, Fallowfield L, et al. Ovarian cancer. Nat Rev Dis Primers. 2016;2:16061. doi:10.1038/nrdp.2016.61

6. Berek JS, Kehoe ST, Kumar L, et al. Cancer of the ovary, fallopian tube, and peritoneum. Int J Gynecol Obstetr. 2018;143:59-78. doi:10.1002/ijgo.12614

7. Coleman RL, Monk BJ, Sood AK, et al. Latest research and treatment of advanced-stage epithelial ovarian cancer. Nat Rev Clin Oncol. 2013;10(4):211. doi:10.1038/nrclinonc.2013.5

8. Deng L, Wang Y, Lu W, et al. Apatinib treatment combined with chemotherapy for advanced epithelial ovarian cancer: a case report. Onco Targets Ther. 2017;10:1521. doi:10.2147/OTT.S126471

9. Gray H, Benigno B, Berek J, et al. Progression-free and overall survival in ovarian cancer patients treated with CVac, a mucin 1 dendritic cell therapy in a randomized Phase 2 trial. $J$ Immunother Cancer. 2016;4(1):34. doi:10.1186/s40425-016-0137-x

10. Fenglian X, Degn F, Long Y, et al. Clinical effect of sevoflurane and propofol anesthesia on hemorrheology in elderly patients with colon cancer radical surgery. Chin J Biochem Pharm. 2017;37(3):240-242.

11. Oh C-S, Lee J, Yoon T-G, et al. Effect of equipotent doses of propofol versus sevoflurane anesthesia on regulatory $\mathrm{T}$ cells after breast cancer surgery. Anesthesiology. 2018;129(5):921-931. doi:10. 1097/ALN.0000000000002382

12. Wang L, Wang T, Gu J-Q, et al. Volatile anesthetic sevoflurane suppresses lung cancer cells and miRNA interference in lung cancer cells. Onco Targets Ther. 2018;11:5689. doi:10.2147/OTT. S171672

13. Ding J, Zhang L, Zeng S, et al. Clinically relevant concentration of sevoflurane suppresses cervical cancer growth and migration through targeting multiple oncogenic pathways. Biochem Biophys Res Commun. 2019. doi:10.1016/j.bbrc.2019.05.082

14. Gao C, Shen J, Meng Z-X, et al. Sevoflurane inhibits glioma cells proliferation and metastasis through miRNA-124-3p/ROCK1 axis. Pathol Oncol Res. 2019;1-8.

15. Yang X, Zheng YT, Rong W. Sevoflurane induces apoptosis and inhibits the growth and motility of colon cancer in vitro and in vivo via inactivating Ras/Raf/MEK/ERK signaling. Life Sci. 2019;116916. 
16. Siegel R, Ma J, Zou Z, et al. Cancer statistics, 2014. CA Cancer J Clin. 2014;64(1):9-29. doi:10.3322/caac.21208

17. Narod S. Can advanced-stage ovarian cancer be cured? Nat Rev Clin Oncol. 2016;13(4):255. doi:10.1038/nrclinonc.2015.224

18. Flanagan JM, Wilson A, Koo C, et al. Platinum-based chemotherapy induces methylation changes in blood DNA associated with overall survival in patients with ovarian cancer. Clin Cancer Res. 2017;23 (9):2213-2222. doi:10.1158/1078-0432.CCR-16-1754

19. Deraco M, Kusamura S, Virzì S, et al. Cytoreductive surgery and hyperthermic intraperitoneal chemotherapy as upfront therapy for advanced epithelial ovarian cancer: multi-institutional phase-II trial. Gynecol Oncol. 2011;122(2):215-220. doi:10.1016/j.ygyno.2011.05. 004

20. Zou S, Cao N, Cheng D, et al. Enhanced apoptosis of ovarian cancer cells via nanocarrier-mediated codelivery of siRNA and doxorubicin. Int J Nanomedicine. 2012;7:3823. doi:10.2147/IJN.S30631

21. Fu Y, Zhang Q, Wang X, et al. Antidiabetic drug metformin mitigates ovarian cancer SKOV3 cell growth by triggering G2/M cell cycle arrest and inhibition of m-TOR/PI3K/Akt signaling pathway. Eur Rev Med Pharmacol Sci. 2017;21(5):1169-1175.

22. Xu Y, Wang S, Chan HF, et al. Dihydromyricetin induces apoptosis and reverses drug resistance in ovarian cancer cells by p53-mediated downregulation of survivin. Sci Rep. 2017;7:46060. doi:10.1038/ srep46060

23. Rhondali O, Pouyau A, Mahr A, et al. Sevoflurane anesthesia and brain perfusion. Pediatr Anesth. 2015;25(2):180-185. doi:10.1111/ pan.2015.25.issue-2

24. Liu J, Yang L, Guo X, et al. Sevoflurane suppresses proliferation by upregulating microRNA-203 in breast cancer cells. Mol Med Rep. 2018;18(1):455-460. doi:10.3892/mmr.2018.8949

25. Yang Y, Hu R, Yan J, et al. Sevoflurane inhibits the malignant potential of head and neck squamous cell carcinoma via activating the hypoxia-inducible factor- $1 \alpha$ signaling pathway in vitro. Int $J \mathrm{Mol}$ Med. 2018;41(2):995-1002. doi:10.3892/ijmm.2017.3306
26. Liang $\mathrm{H}, \mathrm{Gu} \mathrm{M}-\mathrm{N}$, Yang C-X. Sevoflurane inhibits proliferation, induces apoptosis, and blocks cell cycle progression of lung carcinoma cells. Asian Pac j Cancer Prev. 2011;12(12):3415-3420.

27. Iwasaki M, Zhao H, Jaffer $\mathrm{T}$, et al. Volatile anaesthetics enhance the metastasis related cellular signalling including CXCR2 of ovarian cancer cells. Oncotarget. 2016;7(18):26042. doi:10.18632/oncotarget.v7i18

28. Choe KN, Moldovan GL. Forging ahead through darkness: PCNA, still the principal conductor at the replication fork. Mol Cell. 2017;65 (3):380-392. doi:10.1016/j.molcel.2016.12.020

29. Pu X, Storr SJ, Zhang Y, et al. Caspase-3 and caspase-8 expression in breast cancer: caspase- 3 is associated with survival. Apoptosis. 2017;22(3):1-12. doi:10.1007/s10495-016-1323-5

30. Xu Y, Qin L, Sun T, et al. Twist1 promotes breast cancer invasion and metastasis by silencing Foxal expression. Oncogene. 2017;36 (8):1157-1166. doi:10.1038/onc.2016.286

31. Olaso E, Ikeda K, Eng FJ, et al. DDR2 receptor promotes MMP-2mediated proliferation and invasion by hepatic stellate cells. J Clin Invest. 2001;108(9):1369. doi:10.1172/JCI200112373

32. Ramos-Desimone N, Hahn-Dantona E, Sipley J, et al. Activation of matrix metalloproteinase-9 (MMP-9) via a converging plasmin/stromelysin-1 cascade enhances tumor cell invasion. $J$ Biol Chem. 1999;274(19):13066-13076. doi:10.1074/jbc.274.19.13066

33. Sun Y, Liu W-Z, Liu T, et al. Signaling pathway of MAPK/ERK in cell proliferation, differentiation, migration, senescence and apoptosis. J Recept Sig Transd. 2015;35(6):600-604. doi:10.3109/ 10799893.2015.1030412

34. You Z, Liu SP, Du J, et al. Advancements in MAPK signaling pathways and MAPK-targeted therapies for ameloblastoma: a review. J Oral Pathol Med. 2019;48(3):201-205. doi:10.1111/jop.12807

35. Wang $\mathrm{S}$, Wang $\mathrm{M}$, Wang $\mathrm{M}$, et al. Bavachinin induces oxidative damage in HepaRG cells through p38/JNK MAPK pathways. Toxins. 2018;10(4):154. doi:10.3390/toxins 10040154

\section{Publish your work in this journal}

Drug Design, Development and Therapy is an international, peerreviewed open-access journal that spans the spectrum of drug design and development through to clinical applications. Clinical outcomes, patient safety, and programs for the development and effective, safe, and sustained use of medicines are a feature of the journal, which has also been accepted for indexing on PubMed Central. The manuscript management system is completely online and includes a very quick and fair peer-review system, which is all easy to use. Visit http://www. dovepress.com/testimonials.php to read real quotes from published authors. 